\title{
Cytopathologic identification of circulating tumor cells (CTCs) in breast cancer: Application of size-based enrichment
}

\author{
Mohamed Kamal ${ }^{1}$, Macall Leslie' ${ }^{1}$ Crista Horton ${ }^{3}$, Natalie Hills ${ }^{3}$, Rachel Davis ${ }^{3}$, Richard Nguyen ${ }^{3}$, Mohammad Razaq ${ }^{1}$, Katherine Moxley ${ }^{4}$, \\ Paul Hofman ${ }^{5}$, Roy Zhang ${ }^{2 *}$ and Takemi Tanaka ${ }^{1,2 *}$ \\ ${ }^{1}$ University of Oklahoma Health Sciences Center, Stephenson Cancer Center, 975 NE, 10th, Oklahoma City, OK 73104, USA \\ ${ }^{2}$ University of Oklahoma Health Sciences Center, College of Medicine, Dept. of Pathology, 940 SL Young Blvd, Oklahoma City, OK 73104, USA \\ ${ }^{3}$ University of Oklahoma Health Sciences Center, College of Medicine, Oklahoma City, OK 73104, USA \\ ${ }^{4}$ University of Oklahoma Health Sciences Center, Stephenson Cancer Center, Dept. of Gynecologic Oncology, 800 NE, 10th, Oklahoma City, OK 73104, USA \\ ${ }^{5}$ University Côte d'Azur, Laboratory of Clinical and Experimental Pathology, BB-0033-00025, FHU OncoAge, Pasteur Hospital, BP69, 06001 Nice cedex 01, France
}

\begin{abstract}
Circulating tumor cells (CTCs) are indicative of metastatic disease in multiple types of solid tumors. Technologic advances in CTC enrichment have yielded profound variability in both quantity and phenotypic characteristics of CTCs. While size-based exclusion methods have improved the sensitivity of CTC capture, their diminished specificity requires subsequent robust cytopathologic identification of CTCs. In this study, we compared CTC counts from Isolation by Size of Epithelial/Trophoblastic Tumor cells $\left(\right.$ ISET $\left.^{\circledR}\right)$ filters sequentially stained by May-Grünwald/Giemsa (MGG), immunocytochemistry (ICC)/hematoxylin, and ICC/hematoxylin/eosin, followed by corresponding CTC criteria. An immune and endothelial cell cocktail of CD45/CD11b/CD31 antibodies adequately ruled out immune and endothelial cells, yet a substantial number of atypical morphologies with nuclear irregularity (i.e., circulating non-hematological/endothelial cells; $\mathrm{CNHCs}$ ) were detected in both breast cancer cases and non-cancerous controls following hematoxylin nuclear counterstain. Cytoplasmic staining with eosin, significantly diminished CNHC counts. In conclusion, detection of CTCs from ISET filters using chromogenic ICC is feasible in conjunction with identification criteria of nuclear irregularity, negative reactivity to immune and endothelial cell markers, and presentation of visible cytoplasm.
\end{abstract}

\section{Introduction}

Breast cancer is the most commonly diagnosed malignancy as well as a leading cause of cancer-related mortality among women globally [1]. While prevention of recurrence is the primary goal for early-stage breast cancer, palliative care to alleviate symptoms and extension of survival time become the primary focus in metastatic disease. Therefore, accurate prediction of prognosis, as well as early detection of metastatic disease, are crucial for clinical management.

Circulating tumor cells (CTCs) have been highlighted as a "liquid biopsy," enabling non-invasive longitudinal disease monitoring [2,3]. CTCs are mediators of hematogenous metastasis, through which viable cancer cells from the primary tumor enter the circulation and infiltrate distant organs. Thus, the prevalence of CTCs is indicative of metastatic disease and associated with poorer clinical outcomes in multiple types of malignancies [4-6]. The first large, multi-institutional clinical study by Cristofanilli, et al. concluded that patients with $\geq 5$ CTCs in $7.5 \mathrm{ml}$ of pre-treatment blood have shorter progression-free survival compared to those with less than 5 CTCs [2]. Numerous studies have followed, investigating the clinical validity of CTCs in the prediction of prognosis [3,7-9] and therapy response $[3,10,11]$ Given the conceptual validity of CTCs as a prognostic marker, a number of new technologies for the detection of CTCs have emerged [12].

In circulation, CTCs represent only a minute fraction of a large number of circulating cells (immune, red blood, nontumoral epithelial cells, endothelial cells, and fibroblasts) [13-18]; therefore, high sensitivity and specificity are prerequisites for CTC isolation and characterization [13-15]. CellSearch ${ }^{\otimes}$ was the first FDA-approved semi-automated CTC detection system, in which CTCs are enriched based on the expression of epithelial marker (EpCAM), and subsequent identification based on positive expression of cytokeratins (CK), negative expression of immune cell marker (CD45), and a nuclear/cytosol ratio $>50 \%$ [19]. While epithelial properties (i.e., EpCAM expression) are the broadly adopted basis for CTC enrichment strategies [19], loss of epithelial marker expression during epithelial-mesenchymal transition (EMT), presents a plausible concern for inadvertently lower CTC counts $[20,21]$. To address this, new enrichment strategies that exploit the differential physio-chemical properties of CTCs have emerged [22,23].

*Correspondence to: Takemi Tanaka, Ph.D., Associate Professor, University of Oklahoma Health Sciences Center, School of Medicine, Dept. of Pathology, Stephenson Cancer Center at 975 NE 10th, BRC-W, Rm 1415, Oklahoma City, OK, 73104, USA, Tel: Office (405)-271-8260,E-mail: takemi-tanaka@ouhsc.edu

Roy Zhang, MD., Associate Professor, University of Oklahoma Health Sciences Center, School of Medicine, Dept. of Pathology, 940 SL Young Blvd, Oklahoma City, OK 73104, USA, Tel: Office (405)-271-1794, E-mail: roy-zhang@ouhsc.edu

Key words: circulating tumor cells, detection, characterization, cytology

Received: December 06, 2019; Accepted: December 12, 2019; Published: December 16, 2019 
Common morphologic abnormalities of carcinoma cells (e.g., enlarged nucleus and high nuclear density) compared to normal epithelial and immune cells provide the theoretic basis of physicochemical propertybased CTC enrichment strategies [24-27].

A size-based exclusion principle for CTC enrichment permits sensitive capture of CTCs on a porous filter ( 6.5 to $8.0 \mu \mathrm{m}$ pores) with minimal biological bias (i.e., exclusion of EpCAM negative cells) [26,27]. Different technologies such as Isolation by Size of Tumor cells (ISET ${ }^{\circ}$ ) [28] and Screencells substantially increase the detection sensitivity, yet a reduction in specificity demands cytopathologic identification of CTCs $[25,29]$. Previous works have used ISET (a size-based CTC enrichment method) to conduct blinded, multicenter studies evaluating the feasibility of CTC identification by May-Grünwald/Giemsa (MGG) staining and the following cytopathologic criteria: non-hematologic cells with visible cytoplasm having at least 4 of the following featuresirregular nuclei, anisonucleosis (ratio $>0.5$ ), high nuclear/cytoplasmic ratio, nuclei larger than $24 \mu \mathrm{m}$, or the presence of tridimensional sheets $[30,31]$. Based on these criteria, CTCs were only detected in the blood of patients with malignant disease and were absent in healthy subjects [32]. Another study showed the superior sensitivity of CellSearch for the detection of CTCs in non-small cell lung cancer patients [33]. Despite the high sensitivity of ISET, CTC enumeration methods have not yet been standardized, yielding discordant results. In this study, we compared CTC counts through sequential staining of ISET filters with MGG, Immunocytochemistry (ICC)/hematoxylin and ICC/ hematoxylin/eosin, accompanied by corresponding cytomorphological criteria of CTCs to determine the optimal process for accurate CTC detection.

\section{Methods}

Cases: Women newly diagnosed with breast cancer (Stage I-IV) from 2014 to 2016 (19 subjects) and non-cancerous individuals (5 subjects) were enrolled ( 24 individuals total). Breast cancer patients with previous history of any malignancy or synchronous cancer were excluded from the study. All enrollees were consented for study as approved by the IRB committee of the University of Oklahoma Health Science Center and provided written informed consent. Peripheral blood $(10 \mathrm{~mL})$ was drawn before initiation of therapy. Blood was collected in a Vacutainer EDTA tube (BD Biosciences, Franklin Lakes, NJ, USA), delivered to the laboratory at ambient temperature, and processed within 2 hours of blood draw.

CTC enrichment: CTCs were isolated from $10 \mathrm{~mL}$ of peripheral blood following the protocol described by ISET $^{\oplus}$ (RareCells, Paris, France). Peripheral blood was mixed with formaldehyde and sodium hydroxide containing RareCells Buffer solution ( $\mathrm{pH}$ 7.2) for simultaneous red blood cell rupture and formalin fixation for $10 \mathrm{~min}$ at room temperature under constant gentle reciprocal shaking and then filtered through a polycarbonate filter with a calibrated pore size of $8 \mu \mathrm{m}$ under negative pressure $(<10 \mathrm{kPa})$. The filter was gently washed with phosphate buffered saline (PBS) followed by DI water, then air-dried.

May-Grünwald/Giemsa (MGG) staining of ISET filter: The stored filter was immersed in PBS for 1 hour to hydrate, then in a MayGrünwald solution (Biolyon, France) for 5 min followed by 1:1 dilution of May-Grünwald solution for another $5 \mathrm{~min}$ at room temperature. The filter was then immersed in 1:10 dilution of Giemsa (Biolyon, France) for $40 \mathrm{~min}$. After a brief wash with PBS and DI water, the dried filter was subjected to cytopathologic analysis under a light microscope. Filters with signs of hemolysis as well as clots were removed from the study.
Immunocytochemistry (ICC): The MGG stained ISET filter was immersed in $0.5 \%$ acetic acid in $70 \%$ ethanol for $5 \mathrm{~min}$. Antigen retrieval was performed at $60^{\circ} \mathrm{C}$ in Envision FLEX Target Retrieval Solution (high $\mathrm{pH}$, Dako, Santa Clara, CA) for $10 \mathrm{~min}$. The filter was then incubated for 3 min to block endogenous peroxidase (Peroxidazed1, Biocare Medical, Concord, CA) followed by 2 min incubation in a blocking solution (Background Sniper, Biocare Medical). For enumeration of CTCs, the filter was incubated with an immune and endothelial cell [34] cocktail including anti-mouse monoclonal antibodies (BD Biosciences) for CD45 (1:150), CD11b (1:100), and CD31 (1:100) overnight at room temperature. After a brief wash with PBS, the filter was incubated with secondary AP-polymer conjugated $\mathrm{MACH} 2$ anti-mouse IgG (Biocare Medical) for $30 \mathrm{~min}$ at room temperature, then visualized by Vulcan Fast Red (Biocare Medical) until the desired color appeared. Finally, the filter was counterstained with Harris Modified Hematoxylin (Fisher Chemical, Pittsburgh, PA), air-dried, and subjected to pathologic evaluation. The same filter was next subjected to further staining with Eosin (Ricca chemical, Arlington, TX). The filter was analyzed under a light microscope using Leica Application Suite Version 4.7 (Leica). For fluorescent staining, unstained filters were briefly immersed in PBS-Tween ( $0.01 \%$ ) for $3 \mathrm{~min}$, incubated with Hoechst 33342 for $5 \mathrm{~min}$, and then eosin for $1 \mathrm{~min}$. The cells were imaged by a fluorescent microscope (Leica).

CTC criteria: Circulating non-hematological/endothelial cells (CNHCs) with or without malignant features were enumerated from 8 spots per subject, equivalent to $8 \mathrm{~mL}$ blood. CTC criteria for the MGG stained filter were visible cytoplasm with at least 4 of the following: nucleus/cytosol ratio $>0.5$, nuclei larger than a 3 -calibrated pore size $(>24 \mu \mathrm{m})$, irregular nuclei, the presence of tridimensional sheets, or anisonucleosis $^{32}$. In ICC/hematoxylin, CTC criteria were adapted from previously reported studies as a CNHC with no reactivity to $\mathrm{CD} 45 / \mathrm{CD} 11 \mathrm{~b} / \mathrm{CD} 31$, with nuclear irregularity including nuclear/ cytoplasmic ratio $>0.5$, negative, and nuclear irregularities (size $>16$ $\mu \mathrm{m}$, dense nucleus, nuclear indentation) [24,35]. CTC criteria for ICC/ hematoxylin/eosin was CNHCs with a nuclear/cytoplasmic ratio $>0.5$, and the presence of nuclear irregularities (size $>16 \mu \mathrm{m}$, dense nucleus, nuclear indentation) as well as intact plasma membrane. The longest diameter of the nucleus was measured using scale function. The term "malignant CNHCs" used in this study is equivalent to CTCs.

\section{Results}

\section{Consecutive CTC counting using different staining methods}

To compare the accuracy of CTC detection, ISET filters were consecutively stained with 3 different methods- 1) MGG cytology staining, 2) ICC for CD45/CD11b/CD31 and hematoxylin staining, and 3) ICC for CD45/CD11b/CD31, hematoxylin, and cytoplasmic staining with eosin. CTCs were enumerated sequentially from the same filter according to previously reported CTC criteria [24,32,35]. A total of 19 invasive ductal breast carcinoma cases (Stage I-IV) and 5 non-cancerous controls were included in the study. Whole blood $(10 \mathrm{~mL})$ was processed and filtered within 2 hours from the blood draw, and resulting ISET filters were used for consecutive staining and cytopathologic evaluation.

Following filtration, MGG staining detected approximately 150 cells remaining on each spot of the ISET filters (ranging from 50250 cells per spot). Two cases were removed from the study due to significant blood clots and hemolysis. Lymphocytes and neutrophils were the predominant cell populations; the majority of lymphocytes were normal in morphologic appearance (Supplementary Figure 1a). 
Lymphocytes showed small rounded nuclei with uniform chromatin and scanty cytoplasm on the ISET filter. Although rare, lymphocytes with a slightly larger nucleus and increased cytoplasm indicate the presence of reactive ones (Supplementary Figure 1b). Various sizes of cells with indented nuclei, light chromatin, and an intermediate amount of cytoplasm with rare, small vacuoles were presumably monocytes (Supplementary Figure 1c and d). Eosinophils were also present, albeit fewer, and showed classic bi-lobed nucleus and intermediate cytoplasm with red granules (Supplementary Figure 1e). Neutrophils were multilobed with an intermediate amount of cytoplasm and pale granules in our staining (Supplementary Figure 1f). Aggregated lobes of nuclei were slightly larger than the size of lymphocytes. No residual red blood cells were detected on the ISET filter. Apart from the aforementioned immune cells, abnormal cytomorphology was also noted as a single, duplet, triplet, or larger clusters (Figure $1 \mathrm{~g}$ to $\mathrm{k}$ ), whose sizes were at least 2-3 times the greatest dimension of lymphocytes. MGG staining and corresponding cytology [30,31] detected a total of 15 CNHCs of malignancy in 2 out of 19 cases, one of which had a cluster of 10 malignant CNHCs. No abnormal morphology was noted in noncancerous cases (Table 1).

MGG stained ISET filters were de-stained and heat retrieved for subsequent ICC with antibody cocktail including CD45/CD11b/CD31 followed by hematoxylin staining for negative identification of CNHCs. Single staining of CD45 displayed a positive reaction in lymphocytes, monocytes, eosinophils, and neutrophils (Supplementary Figure 2a-d). Monocytes showed a range of reactivity to CD45. In contrast, CD11b was widely positive in myeloid cells; but displayed a range of reactivity in lymphocytes (Supplementary Figure 2e-h). Size of monocytes varied significantly, with the presentation of nuclear size $>24 \mu \mathrm{m}$ and nuclear indentation (Supplemental Figure 2i). Although rare, weakly positive reaction to $\mathrm{CD} 31$ was noted in a minor fraction of granulocytes and lymphocytes (Supplementary Figure 2j) as well as platelets (Supplementary Figure 2k). However, no atypical morphology was detected. Thus, the cocktail of CD45/CD11b/CD31 antibodies broadly covered both lymphoid, myeloid, and endothelial cells for negative identification of CNHCs in this study. The rate of cell detachment from the ISET filter throughout the ICC procedure was $4.3 \%$ (ranging from $0.93 \%$ to $14.7 \%, \mathrm{n}=7$ ) when MGG stained ISET filters were subjected to mock ICC with heat retrieval at $90{ }^{\circ} \mathrm{C}$ for $40 \mathrm{~min}$, and the retention of corresponding cells was counted (Supplementary Table 1). Thus, ICC of ISET filters is feasible with minor cell loss, and a CD45/CD11b/ CD31 cocktail successfully detects a broad range of circulating immune cells and endothelial cells, while leaving the remaining circulating cells (CNHCs) of malignancy and uncertainty of malignancy unstained.

Among cells with no reactivity to CD45/CD11b/CD31, hematoxylin counterstaining visualized nuclear irregularities (dense nucleus, nuclear indentation, and rough nuclear surface) within a size range of 16-31 $\mu \mathrm{m}$. According to CTC criteria [24,35], a total of 251 atypical cells were detected from all breast cancer patients. Atypical cells were present across samples of all stage, tumor size, nodal status, histology grade, metastasis, and subtype groups. Consistent with previous reports [33], CNHCs were present in 3 out of 5 non-cancerous cases ranging from 0-12 (Table 1). Confirmatory eosin staining for cell integrity diminished the number of CNHCs detected from 251 to 34 ; with over $200 \mathrm{CNHCs}$ showing no cytoplasmic staining with eosin as depicted in representative images (Figure 1a and b). The resulting $34 \mathrm{CNHCs}$ that displayed malignant features were found in stage IV (4/4), stage III (4/5), and stage II (1/7) cases; demonstrating a trend of higher prevalence in metastatic and more advanced cases.

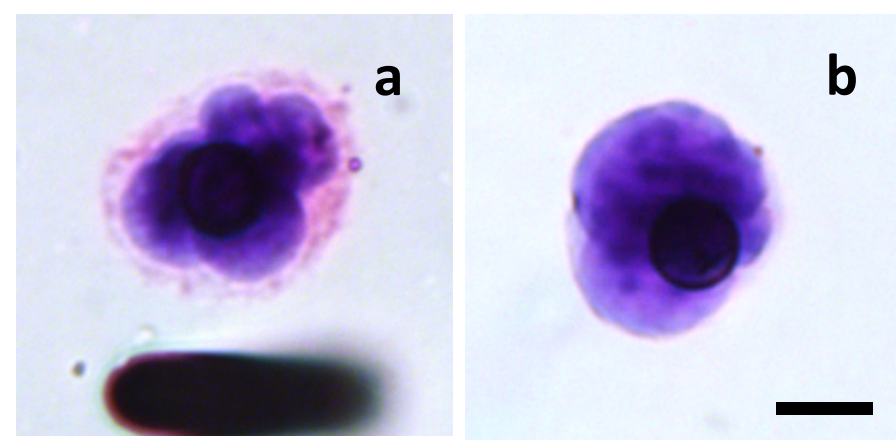

Figure 1. CNHCs with or without positive staining for plasma membrane. CNHCs with no reactivity to $C D 45 / C D 11 b / C D 31$ with nuclear irregularities with (a) or without (b) intact plasma membrane. ISET filters were ICC stained with CD45/CD $11 \mathrm{~b} / \mathrm{CD} 31$ followed by hematoxylin. Following identification of atypical morphologies under microscope, the filter was further subjected to Eosin staining. Images were taken at a final magnification of $\times 630$; scale bar indicates $10 \mu \mathrm{m}$

Table 1. Differential CNHCs counts on ISET filter. CNHCs were counted on ISET filter sequentially by MGG cytology, ICC/hematoxylin, and ICC/hematoxylin/eosin in blood collected from invasive breast cancer and non-cancerous cases

\begin{tabular}{|c|c|c|c|c|c|c|}
\hline \multirow[b]{2}{*}{ Patient No. } & \multirow[b]{2}{*}{ Stage } & \multirow[b]{2}{*}{ Grade } & \multirow[b]{2}{*}{ Subtype } & \multicolumn{3}{|c|}{ CNHC Counts: } \\
\hline & & & & MGG & IHC + HX & $\begin{array}{c}\text { IHC + HX } \\
+ \text { Eosin }\end{array}$ \\
\hline 1 & IV & 3 & $\mathrm{TN}$ & 0 & 6 & 3 \\
\hline 2 & IIA & 3 & LumB & 0 & 5 & 0 \\
\hline 3 & IIIA & 3 & LumB & 0 & 0 & 0 \\
\hline 4 & IIIA & 3 & Her2 & $1^{*}$ & 21 & 3 \\
\hline 5 & IIA & 3 & $\mathrm{TN}$ & 0 & 0 & 0 \\
\hline 6 & IA & 2 & LumA & 0 & 20 & 0 \\
\hline 7 & IV & 2 & LumA & 0 & 10 & 1 \\
\hline 8 & IIA & 3 & LumA & 0 & 27 & 1 \\
\hline 9 & IIIC & 3 & LumA & 5 & 25 & 6 \\
\hline 10 & IIA & 2 & LumA & 0 & 22 & 0 \\
\hline 11 & IV & 3 & LumB & 0 & 25 & 11 \\
\hline 12 & IIB & 3 & Her2 & 0 & 10 & 2 \\
\hline 13 & IIIA & 3 & Her2 & 0 & 12 & 1 \\
\hline 14 & IV & 3 & $\mathrm{TN}$ & 0 & 5 & 2 \\
\hline 15 & IIIA & 2 & LumA & 0 & 33 & 4 \\
\hline 16 & IIA & 3 & $\mathrm{TN}$ & 0 & 16 & 0 \\
\hline 17 & IIA & 3 & TN & 0 & 5 & 0 \\
\hline $18^{\dagger}$ & & & & & 1 & 0 \\
\hline $19^{\dagger}$ & & & & & 0 & 0 \\
\hline $20^{\dagger}$ & & & & & 11 & 0 \\
\hline $21^{\dagger}$ & & & & & 0 & 0 \\
\hline $22^{\dagger}$ & & & & & 12 & 0 \\
\hline
\end{tabular}

*denotes a circulating tumor microemboli (CTM), defined as a cluster of 2 or more cells 'non-cancerous control

All CNHCs detected in non-cancerous controls were excluded due to a lack of cytoplasmic staining. Fluorescent staining also detected nuclear irregularities; some showed the positive cytoplasmic staining with eosin (red fluorescence; Supplementary Figure 3a-c), while it was absent in others (Supplementary Figure 3d and f). Of note, most of malignant CNHCs identified by ICC/hematoxylin/eosin staining had nuclei smaller than $24 \mu \mathrm{m}$. Therefore, CNHCs detected by ICC/ hematoxylin based negative staining and nuclear irregularity contains admixed populations, malignant CNHCs, CNHCs of the uncertainty of malignancy, and nucleus without intact cytoplasm. Thus, these data demonstrated that 1) $24 \mu \mathrm{m}$ is not appropriate cutoff size for breast CTCs, and 2) negative ICC with CD markers and hematoxylin detects a large number of false positives without intact cytoplasm, 3) 
confirmatory cytoplasmic staining is essential to identify structurally intact malignant CNHCs.

\section{Discussion}

Identification of CTCs requires initial enrichment and subsequent validation. Two major differential CTC properties (biological and physiochemical) have been adopted for enrichment as well as detection strategies. Biological properties of CTCs, are primarily exploited for affinity-based capture through interaction between cell surface epithelial markers and their corresponding antibody [19]. Heterogeneity is a hallmark of cancer, and mounting evidence indicates both genetic and phenotypic heterogeneity in CTCs [36-38]. In particular, the loss of epithelial features and gain of mesenchymal properties (EMT) directly impacts CTC counts as well as their clinical significance. Physiochemical properties of CTCs (cell size, density, loss of elasticity, and surface charge) have been adopted as a less biologically biased CTC enrichment strategy [25]. Size-based exclusion methods enrich circulating cells on the porous filters, leaving cells larger than the pores. The main populations of residual cells are lymphocytes and myeloid cells, yet atypical cells including CTCs are also detected. The diversity of residual cell populations necessitates further robust identification of CTCs; thus, differential morphological (size, shape, and nuclear irregularity) or cytochemical (marker expression) characteristics of CTCs over normal counterparts were exploited $[24,32,35,39]$. Despite the sensitive capture of CTCs, subsequent enumeration remains a challenge, partly due to the lack of standardized and automatized methods.

Regardless of enrichment principal (i.e., solid vs. liquid phase, fixation type, readout, etc.), the most broadly adopted CTC criteria are 1) negative reactivity to immune cell marker (CD45), 2) positive reactivity to cytokeratin and other cancer-related surface markers, and 3) morphologic abnormality [19]. Most studies utilize a combination of at least two of the above. Studies have demonstrated that the CTC detection sensitivity of ISET is superior to CellSearch $[24,33,35,39,40]$. Concomitant comparison found that ISET detects a greater number of CTCs as well as CTC positive cases based on the following MGG cytology criteria in non-small cell lung cancer cases [non-hematologic with visible cytoplasm and at least 4 of the following: irregular nuclei, anisonucleosis (ratio $>0.5$ ), high nuclear/cytoplasmic ratio, nuclei larger than $24 \mu \mathrm{m}$, or the presence of tridimensional sheets] [32]. We used the previously established MGG cytology as well as ICC with CD45/ $\mathrm{CD} 11 \mathrm{~b} / \mathrm{CD} 31$ followed by hematoxylin and eosin staining to detect malignant CNHCs $<24 \mu \mathrm{m}$. Currently, there is no consensus on the size of CTCs [35,39,41,42]. Park, et al. reported that the average diameter of CTCs isolated from 16 prostate cancer patients was $7.97 \pm 1.81$ $\mu \mathrm{m}$ when using the CellSearch system [EpCAM+/PanCK+/CD45-/ $\mathrm{DAPI}+$ and size larger than neighboring cells], which were half the size of prostate cancer cell lines $(13.38 \pm 2.54 \mu \mathrm{m})$ [42]. The size of CTC may vary depending on their origin; CTC cutoff size may need to be determined in a primary tumor dependent fashion.

Consistent with a study published by Castle, et al. ICC/hematoxylin staining detected a large number of CNHCs in both breast cancer patients as well as non-cancerous individuals [35]. However, $90.4 \%$ and $100 \%$ of CNHCs detected by ICC/hematoxylin staining alone in breast cancer patients and non-cancerous individuals, respectively, were excluded due to the absence of visible cytoplasm. The presence of such abnormal morphologies becomes apparent when CTCs are enriched by size, negatively differentiated by $\mathrm{CD}$ markers, and counterstained for nucleus; however, such abnormal morphology (naked-nuclei-like) unlikely interferes with the enumeration when CTCs are positively differentiated by surface marker expression such as EpCAM. The origin and cause of naked-nuclei-like structures remain unknown; however, it is unlikely such structures are related to malignancy due to their high prevalence among non-cancerous individuals. Damage to the plasma membrane may cause swelling of the nuclei due to free fluid or dye passage, in turn, yielding a structure resembling carcinomas with large, dense, irregularly shaped nuclei. Alternatively, positive staining may alleviate this issue [43]. In conclusion, size, nuclear irregularity, and negative reactivity to $\mathrm{CD}$ markers alone may not adequately identify CTCs. Additional confirmatory staining for plasma membrane integrity diminishes risk of counting false positives.

\section{Funding statements}

This work was supported by the Presbyterian Health Foundation (T.T.) and Oklahoma Clinical and Translational Resources (K.M.). These funding have no conflict of interest in the publication.

\section{Conflicts of interest}

All authors have no conflict of interest in this publication.

\section{Data availability}

The image data used to support the findings of this study are included in the article and supplementary information file.

\section{References}

1. Ferlay J, Soerjomataram I, Dikshit R, Eser S, Mathers C, et al. (2015) Cancer incidence and mortality worldwide: sources, methods and major patterns in GLOBOCAN 2012. Int $J$ Cancer 136: E359-E386.

2. Cristofanilli M, Budd GT, Ellis MJ, Stopeck A, Matera J, et al. (2004) Circulating tumor cells, disease progression, and survival in metastatic breast cancer. $N$ Engl $J$ Med 351: 781-791.

3. Nakamura S, Yagata H, Ohno S, Yamaguchi H, Iwata H, et al. (2010) Multi-center study evaluating circulating tumor cells as a surrogate for response to treatment and overall survival in metastatic breast cancer. Breast Cancer 17: 199-204.

4. Zhou Y, Bian B, Yuan X, Xie G, Ma Y, et al. (2015) Prognostic value of circulating tumor cells in ovarian cancer: a meta-analysis. PLoS One 10: e0130873.

5. Zhang ZY, Dai ZL, Yin XW, Li SH, Li SP, et al. (2014) Meta-analysis shows that circulating tumor cells including circulating microRNAs are useful to predict the survival of patients with gastric cancer. BMC cancer 14: 773 .

6. Zhang Z, Fan W, Deng Q, Tang S, Wang P, et al. (2017) The prognostic and diagnostic value of circulating tumor cells in bladder cancer and upper tract urothelial carcinoma: a meta-analysis of 30 published studies. Oncotarget 8: 59527-59538.

7. Zhang L, Riethdorf S, Wu G, Wang T, Yang K, et al. (2012) Meta-analysis of the prognostic value of circulating tumor cells in breast cancer. Clin Cancer Res 18: 5701 5710 .

8. Lv Q, Gong L, Zhang T, Ye J, Chai L, et al. (2016) Prognostic value of circulating tumor cells in metastatic breast cancer: a systemic review and meta-analysis. Clin Transl Oncol 18: 322-330.

9. Bidard FC, Belin L, Delaloge S, Lerebours F, Ngo C, et al. (2013) Time-dependen prognostic impact of circulating tumor cells detection in non-metastatic breast cancer: 70-month analysis of the REMAGUS02 study. Int J Breast Cancer 2013: 130470.

10. Pierga JY, Petit T, Lévy C, Ferrero JM, Campone M, et al. (2015) Pathological response and circulating tumor cell count identifies treated HER2+ inflammatory breast cancer patients with excellent prognosis: BEVERLY-2 survival data. Clin Cancer Res 21: 1298-1304.

11. Budd GT, Cristofanilli M, Ellis MJ, Stopeck A, Borden E, et al. (2006) Circulating tumor cells versus imaging - predicting overall survival in metastatic breast cancer. Clin Cancer Res 12: 6403-6409.

12. Kamal M, Razaq W, Leslie M, Adhikari S, Tanaka T (2017) Circulating Tumor Cells in Breast Cancer: A Potential Liquid Biopsy. Breast Cancer: From Biology to Medicine pp: 119. 
13. Joosse SA, Gorges TM, Pantel K (2015) Biology, detection, and clinical implications of circulating tumor cells. EMBO Mol Med 7: 1.

14. Miller MC, Doyle GV, Terstappen LW (2010) Significance of circulating tumor cells detected by the CellSearch system in patients with metastatic breast colorectal and prostate cancer. J Oncol 2010: 617421.

15. Alix-Panabieres C, Schwarzenbach H, Pantel K (2012) Circulating tumor cells and circulating tumor DNA. Annu Rev Med 63: 199-215.

16. Bidard FC, Mathiot C, Degeorges A, Etienne-Grimaldi MC, Delva R, et al. (2010) Clinical value of circulating endothelial cells and circulating tumor cells in metastatic breast cancer patients treated first line with bevacizumab and chemotherapy. Ann Oncol 21: $1765-1771$.

17. Ao Z, Shah SH, Machlin LM, Parajuli R, Miller PC, et al. (2015) Identification of cancer-associated fibroblasts in circulating blood from patients with metastatic breast cancer. Cancer Res 75: 4681-4687.

18. Gross HJ, Verwer B, Houck D, Hoffman RA, Recktenwald D (1995) Model study detecting breast cancer cells in peripheral blood mononuclear cells at frequencies as low as 10 (-7). Proceedings of the National Academy of Sciences 92: 537-541.

19. Witzig TE, Bossy B, Kimlinger T, Roche PC, Ingle JN, et al. (2002) Detection of circulating cytokeratin-positive cells in the blood of breast cancer patients using immunomagnetic enrichment and digital microscopy. Clin Cancer Res 8: 1085-1091.

20. Bednarz-Knoll N, Alix-Panabières C, Pantel K (2012) Plasticity of disseminating cancer cells in patients with epithelial malignancies. Cancer Metastasis Rev 31: 673687.

21. Mostert B, Kraan J, Sieuwerts AM, van der Spoel P, Bolt-de Vries J, et al. (2012) CD49f-based selection of circulating tumor cells (CTCs) improves detection across breast cancer subtypes. Cancer Lett 319: 49-55.

22. Hu XC, Chow LW (2001) Detection of circulating breast cancer cells with multiplemarker RT-PCR assay. Anticancer Res 21: 421-424.

23. Hawes D, Neville AM, Cote RJ (2001) Occult metastasis. Biomedicine \& pharmacotherapy = Biomedecine \& pharmacotherapie 55: 229-242.

24. Farace F, Massard C, Vimond N, Drusch F, Jacques N, et al. (2011) A direct comparison of CellSearch and ISET for circulating tumour-cell detection in patients with metastatic carcinomas. Br J Cancer 105: 847-853.

25. den Toonder J (2011) Circulating tumor cells: the Grand Challenge. Lab on a Chip 11: 375-357.

26. Kim MS, Sim TS, Kim YJ, Kim SS, Jeong H, et al. (2012) SSA-MOA: a novel CTC isolation platform using selective size amplification (SSA) and a multi-obstacle architecture (MOA) filter. Lab Chip 12: 2874-2880

27. Coumans FA, van Dalum G, Beck M, Terstappen LW (2013) Filter characteristics influencing circulating tumor cell enrichment from whole blood. PloS one 8: e61770.

28. Vona G, Sabile A, Louha M, Sitruk V, Romana S, et al. (2000) Isolation by size of epithelial tumor cells: a new method for the immunomorphological and molecular characterization of circulating tumor cells. Am J Pathol 156: 57-63.

29. Moon HS, Kwon K, Kim SI, Han H, Sohn J, et al. (2011) Continuous separation of breast cancer cells from blood samples using multi-orifice flow fractionation (MOFF) and dielectrophoresis (DEP). Lab Chip 11: 1118-1125.
30. Hofman V, Long E, Ilie M, Bonnetaud C, Vignaud JM, et al. (2012) Morphological analysis of circulating tumour cells in patients undergoing surgery for non-small cell lung carcinoma using the isolation by size of epithelial tumour cell (ISET) method. Cytopathology 23: 30-38.

31. Hofman V, Bonnetaud C, Ilie MI, Vielh P, Vignaud JM, et al. (2011) Preoperative circulating tumor cell detection using the isolation by size of epithelial tumor cell method for patients with lung cancer is a new prognostic biomarker. Clin Cancer Res 17: 827-835.

32. Hofman VJ, Ilie MI, Bonnetaud C, Selva E, Long E, et al. (2011) Cytopathologic detection of circulating tumor cells using the isolation by size of epithelial tumor cell method: promises and pitfalls. Am J Clin Pathol 135: 146-156.

33. Hofman V, Ilie MI, Long E, Selva E, Bonnetaud C, et al. (2011) Detection of circulating tumor cells as a prognostic factor in patients undergoing radical surgery for non-smallcell lung carcinoma: comparison of the efficacy of the CellSearch Assay ${ }^{\mathrm{TM}}$ and the isolation by size of epithelial tumor cell method. Int J Cancer 129: 1651-1660.

34. Ilie M, Long E, Hofman V, Selva E, Bonnetaud C, et al. (2014) Clinical value of circulating endothelial cells and of soluble CD146 levels in patients undergoing surgery for non-small cell lung cancer. Br J Cancer 110: 1236-1243.

35. Castle J, Morris K, Pritchard S, Kirwan CC (2017) Challenges in enumeration of CTCs in breast cancer using techniques independent of cytokeratin expression. PloS one 12 e0175647.

36. Theodoropoulos PA, Polioudaki H, Agelaki S, Kallergi G, Saridaki Z, et al. (2010) Circulating tumor cells with a putative stem cell phenotype in peripheral blood of patients with breast cancer. Cancer lett 288: 99-106.

37. Wang J, Cao MG, You CZ, Wang CL, Liu SL, et al. (2012) A preliminary investigation of the relationship between circulating tumor cells and cancer stem cells in patients with breast cancer. Cell Mol Biol (Noisy-le-grand) 58: OL1641-1645.

38. Yu M, Bardia A, Wittner BS, Stott SL, Smas ME, et al. (2013) Circulating breast tumor cells exhibit dynamic changes in epithelial and mesenchymal composition. science 339: $580-584$

39. Khoja L, Backen A, Sloane R, Menasce L, Ryder D, et al. (2012) A pilot study to explore circulating tumour cells in pancreatic cancer as a novel biomarker. Br J Cancer 106: 508-516.

40. Kallergi G, Politaki E, Alkahtani S, Stournaras C, Georgoulias V (2016) Evaluation of isolation methods for circulating tumor cells (CTCs). Cell Physiol Biochem 40: 411419 .

41. Krebs MG, Hou JM, Sloane R, Lancashire L, Priest L, et al. (2012) Analysis of circulating tumor cells in patients with non-small cell lung cancer using epithelial marker-dependent and-independent approaches. J Thorac Oncol 7: 306-315.

42. Park S, Ang RR, Duffy SP, Bazov J, Chi KN, et al. (2014) Morphological differences between circulating tumor cells from prostate cancer patients and cultured prostate cancer cells. PloS one 9: e85264.

43. Hofman VJ, Ilie M, Hofman PM (2016) Detection and characterization of circulating tumor cells in lung cancer: Why and how?. Cancer Cytopathol 124: 380-387.

Copyright: ( $2019 \mathrm{Kamal} \mathrm{M}$. This is an open-access article distributed under the terms of the Creative Commons Attribution License, which permits unrestricted use, distribution, and reproduction in any medium, provided the original author and source are credited. 